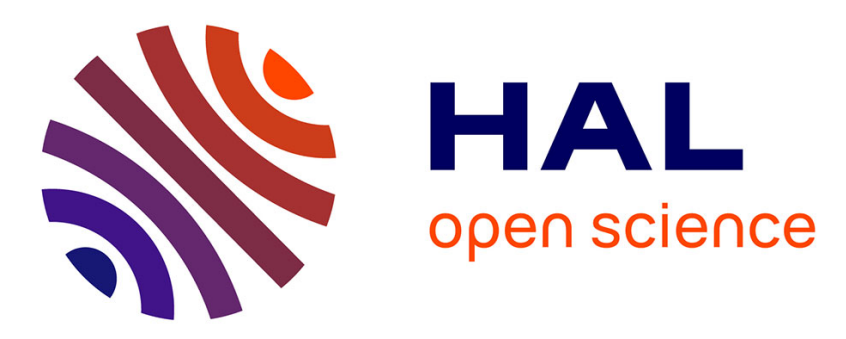

\title{
Nonlinear dynamics and intermittency in a long term copepod time series
}

François G Schmitt, Juan Carlos Molinero, Sylvie Zongo Brizard

\section{To cite this version:}

François G Schmitt, Juan Carlos Molinero, Sylvie Zongo Brizard. Nonlinear dynamics and intermittency in a long term copepod time series. Communications in Nonlinear Science and Numerical Simulation, 2008, 13 (2), pp.407-415. 10.1016/j.cnsns.2006.04.005 . hal-00264838

\section{HAL Id: hal-00264838 \\ https://hal.science/hal-00264838}

Submitted on 18 Mar 2008

HAL is a multi-disciplinary open access archive for the deposit and dissemination of scientific research documents, whether they are published or not. The documents may come from teaching and research institutions in France or abroad, or from public or private research centers.
L'archive ouverte pluridisciplinaire HAL, est destinée au dépôt et à la diffusion de documents scientifiques de niveau recherche, publiés ou non, émanant des établissements d'enseignement et de recherche français ou étrangers, des laboratoires publics ou privés. 


\title{
Nonlinear dynamics and intermittency in a long term copepod time series
}

\author{
François G. Schmitt* \\ CNRS, FRE 2816 ELICO, Wimereux Marine Station, University of Lille 1 \\ 28 av. Foch, 62930 Wimereux, France \\ Juan Carlos Molinero \\ INRA Thonon, France \\ Sylvie Zongo Brizard \\ Wimereux Marine Station, France
}

\begin{abstract}
We consider the nonlinear dynamics of a long-term copepod (small crustaceans) time series sampled weekly in the Mediterranean sea from 1967 to 1992. Such population dynamics display a high variability that we consider here in an interdisciplinary study, using tools borrowed from the field of statistical physics. We analyse the extreme events of male and female abundances, and of the total population, and show that they both have heavy tailed probability density functions (pdf). We provide hyperbolic fits of the form $p(x) \sim 1 / x^{\mu+1}$, and estimate the value fo $\mu$ using Hill's estimator. We then study the ratio of male to female abundances, compared to the female abundances. Using conditional probability density functions and conditional averages, we show that this ratio is independent of the female density, when the latter is larger than a given threshold. This property is very useful for modelisation. We also consider the product of male to female abundances, which can be ecologically related to the encounters. We show that this product is extremely intermittent, and link its pdf to the female pdf.
\end{abstract}

Key words: Biological time series, Extreme events, Intermittency, Nonlinear dynamics

Tel.: +33-321-992935; Fax.: +33-321-992901

E-mail address: francois.schmitt@univ-lille1.fr

F.G. Schmitt, J.C. Molilnero, S. Zongo Brizard. Nonlinear dynamics and intermittency in a long term copepod time series, Comm. Nonlin. Sc. Num. Sim. 13 (2008) 407-415 


\section{Introduction}

A pervasive feature of environmental and geophysical fields is their high variability at many different time and space scales. Given these external constraints, population dynamics is also characterized by a high variability over time or space[1-4]. While many studies in population ecology have focused on average evolutions over time, or considered mean fluctuations, some of them have been concerned by the modelization of extremes [5-8,10]. Extremes are ecologically important for many reasons: high numbers of a species correspond to an economic threat, and to a higher ecological pressure on preys and competitors, but also a higher availability for predators. In the marine ecology framework, phytoplankton blooms are extreme densities, and may induce ecological disturbances. That is the case of harmful algal blooms (HABs) whose occurrence may affect higher forms of life such as zooplankton, shellfish, fish, and subsequently may constitute health risk for human. Other examples may encompass benefits for human, such as extreme densities of crustacean zooplankton populations which provide favorable conditions for important commercial fish, such as cod, sardine and anchovy. We study here the dynamics of a species belonging to crustacean zooplankton: the planktonic copepod Centropages typicus.

In ocean ecosystems planktonic copepods constitute the majority of the mesozooplankton biomass. Their secondary production supports most of the food webs in the open sea directly affecting pelagic fish populations and the biological pump of carbon into the deep ocean [9]. Moreover, these organisms have a typical size of the order of milimeters, a fast life cycle ( $\sim$ one month) and are usually considered to be deeply adapted to their turbulent environment, while being also sentitive to variabilities in the environmental properties, such as temperature, turbidity, turbulence intensity.

We analyse here a long-term data base of Centrapages typicus abundance, sampled every week from 1967 to 1992, focusing on the nonlinear dynamics of the male and female populations, and their extremes. The next section presents the data. Afterwards we analyse extreme events of male and female population abundances in the following section. In section 3 we focus on the ratio male to female, and on its statistical dependence on female population time series. This section provides many new results that can be used directly to model the dynamics of its population. We conclude in the last section. 


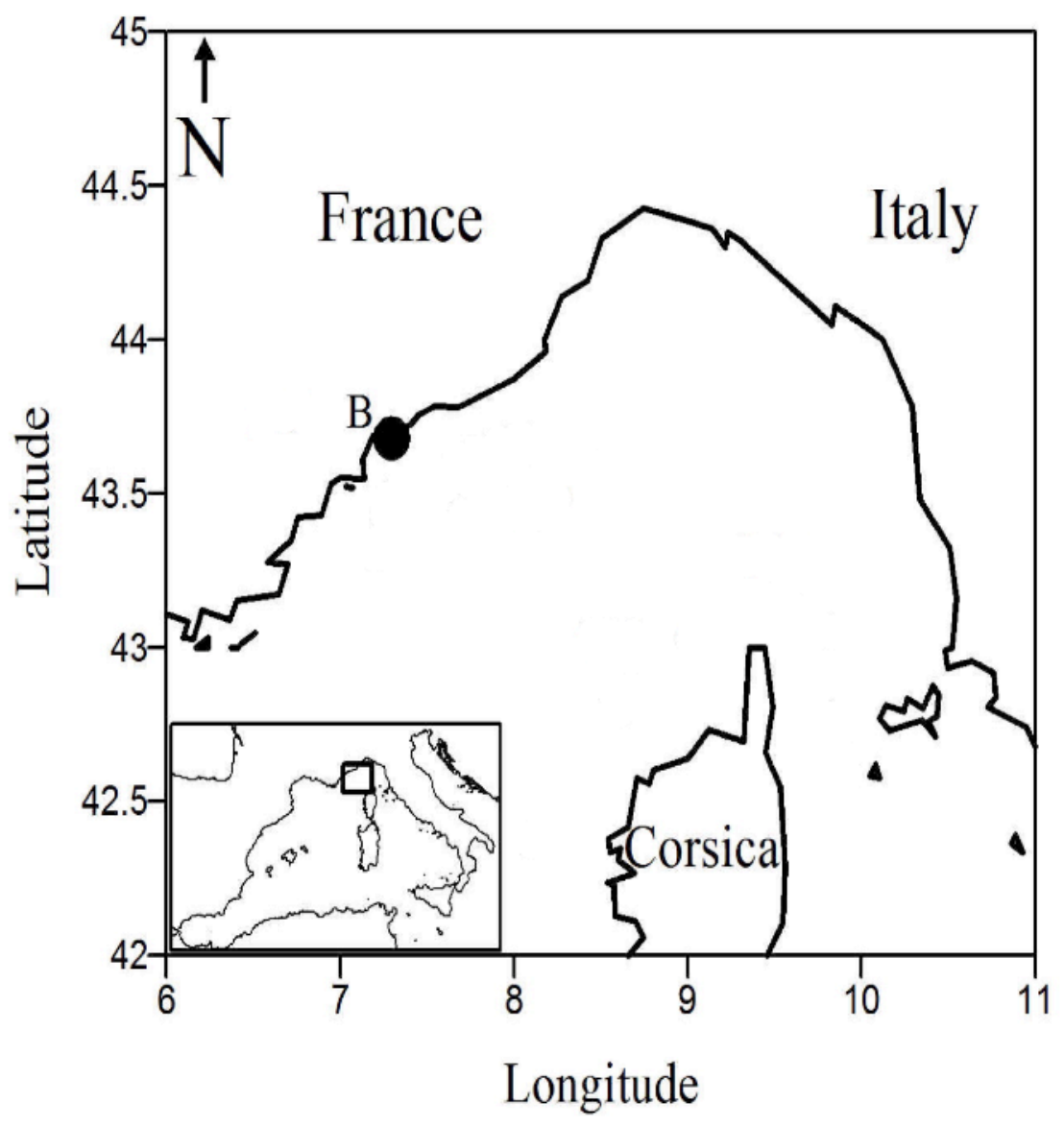

Fig. 1. Map of the western Mediterranean sea (insert) and a zoom to the South East mediterranean coast of France, showing the sampling position ( $B$ in the map, which is located in the Villefranche bay, near Nice.

\section{Presentation of the data base}

The Villefranche Bay is situated in the Ligurian Sea, Northwestern Mediterranean (Fig 1). In the mouth of the bay, a monitoring of plankton variability has been carried out by the Oceanographic Observatory of Villefranche, France (sampling station Pt B located at 43 deg 41' N; 7 deg 19' E) since January 1967. During daylight, plankton sampling was carried out by vertical hauls using a Juday-Bogorov net (mouth aperture $0.5 \mathrm{~m}$, filtering length of $1.80 \mathrm{~m}$ and mesh size of $330 \mu \mathrm{m})$ from the bottom to the surface $(0-75 \mathrm{~m})$. Counts of Centropages typicus adults (males and females) were made under a dissecting microscope. Data we report correspond to the period January 1967 to December 1992. The resulting time series for male and female abundance are shown in Fig. 2: huge fluctuations are clearly visible, indicating the intermittency properties of the time series. 


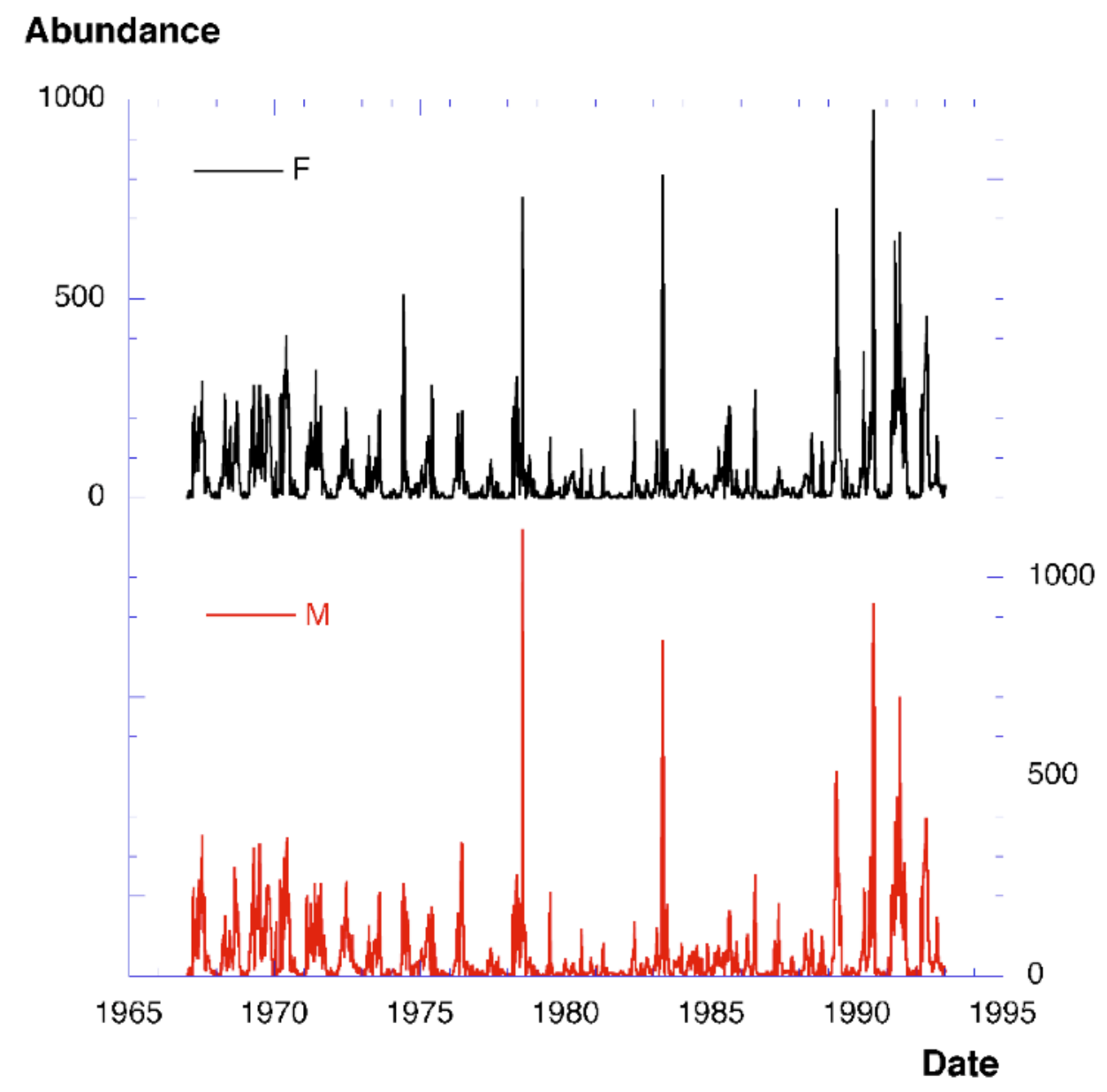

Fig. 2. Times series being analyzed here: females (F) and males $(\mathrm{M})$ in ind. per cubic meters. Huge fluctuations in population densities is clearly visible.

\section{Extreme events}

The probability density function of male, female and total abundance time series is displayed in Fig. 3. Since we focus here on extremes, these pdfs are represented in log-log plots, emphasizing the tail behaviour. They have a tail of the form:

$$
p(X=x)=\frac{C_{0}}{x^{\mu+1}} \quad ; x \gg 1
$$

where $C_{0}$ is a constant, $X$ is the time series, $x$ a threshold values and $\mu>0$ a parameter characterizing the power-law tail behaviour. Such distributions have been given many names in the literature, depending on the fields, called "power-laws", "hyperbolic distributions", "Pareto". In the mathematical theory of extreme events [11], these laws correspond to "type II" and are sometimes called "Frechet laws". Such tail behaviour is much heavier than Gaussian or even lognormal distributions, indicating that extreme events are much more frequent. Power-law distributions have been found in many applications in the 


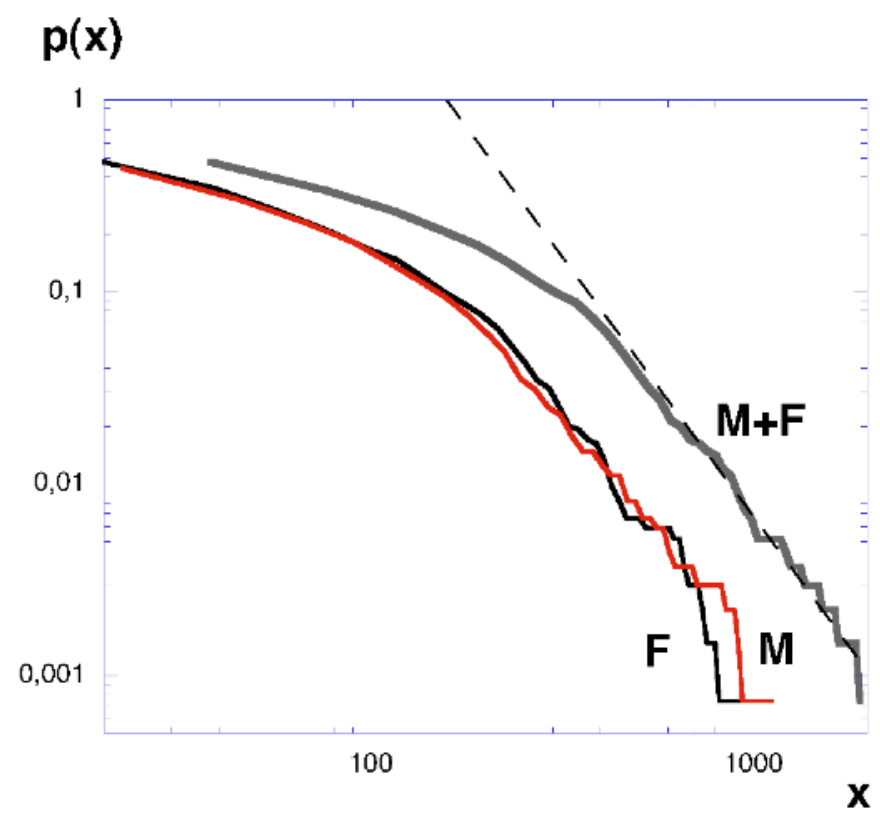

Fig. 3. The pdf of male (M), female $(\mathrm{F})$ and total population $(\mathrm{M}+\mathrm{F})$ abundances, in log-log plot. Such display emphasizes the heavy tails of experimental data. The dotted straight line corresponds to a hyperbolic probability density with an analytical expression of the form $x^{-\mu-1}$, with $\mu=2.5$.

fields of natural or human sciences: for general reviews, see $[12,13]$. Hereafter we denote such tail behaviour as power-law or hyperbolic distributions.

The value of the parameter $\mu$ is important to characterize such distribution. The smaller the parameter, the more intermittent is the time series. If the tail provided by Eq.(1) is valid without upper bound, moments of order $\mu$, diverge:

$$
\left\langle X^{\mu}\right\rangle=\int x^{\mu} p(x) d x \geq C_{0} \int \frac{d x}{x} \rightarrow \infty
$$

The parameter $\mu$ is then a critical order of divergence of moments. When $\mu \leq 2$, the variance of the process diverges, and when $\mu \leq 1$ the mean is not defined. The value of $\mu$ can be provided using an estimator [14-16]. A classical estimator is Hill's estimator [14], defined as:

$$
\mu(k)=\frac{k}{\sum_{i=1}^{k} \log \frac{X_{[i]}}{X_{[k+1]}}}
$$

where the original time series has been ordered, the result denoted $X_{[i]}$ :

$$
X_{[1]} \geq X_{[2]} \geq X_{[3]} \ldots \geq X_{[k]} \cdots \geq X_{[n]}
$$

This estimator corresponds to a mean estimation of the slope of the last $k$ points of the pdf. When $k$ is too small, a scatter is expected on the values of 


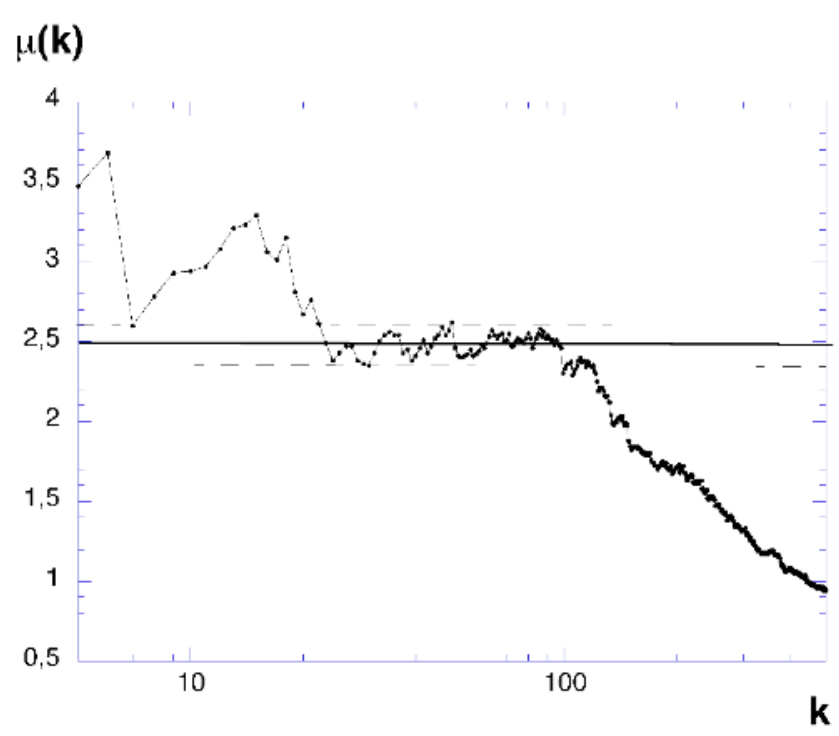

Fig. 4. Hill estimator applied to the total adult population of C. typicus: for small values of $k$, the estimator fluctuates due to poor amount of data; for a medium range of $k$ values, the estimator is very stable, providing a value of $\mu=2.5 \pm 0.05$. For larger values of $k$, the estimator reaches values that do not belong to the hyperbolic tail, and hence it decreases.

$\mu(k)$; for larger values, the estimated value of $\mu$ is given by the plateau reached by the estimator $\mu(k)$. When the hyperbolic law is valid only for extreme events, the estimator will fall down past a given threshold.This estimator is applied on the total population $(M+F$ in Fig. 3) in Fig. 4: a plateau is reached for a range of values of $k$. For these values, we have the following estimation: $\mu=2.5 \pm 0.05$. This value is quite small; it indicates that the variance of the population is defined, but theoretically, its skewness diverges. In practice, this means that the skewness estimated is a value that depends on the number of realizations taken into account: the larger the number of realizations, the larger the numerical estimation of the skewness (see [17] for a discussion in another context). Such fit of the pdf is also interesting for the purpose of modelling of population dynamics. In the following we discuss the male-female dynamics in this framework.

\section{Male-female dynamics}

\subsection{Male to female ratio}

We study here the correspondances between male and female abundances. First, Fig. 5 represents as a scatter plot the female data versus the male data: 


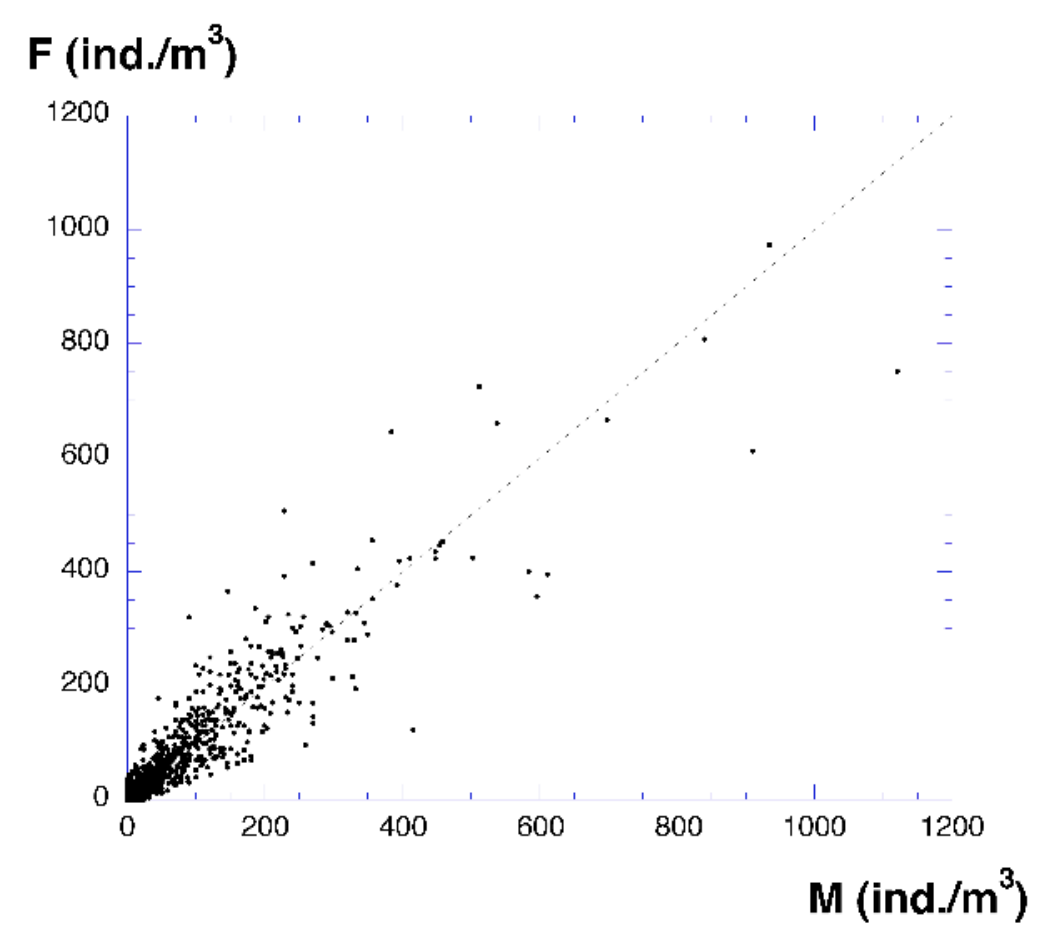

Fig. 5. A scatter plot representing females $(\mathrm{F})$ versus males $(\mathrm{M})$, showing that there is no obvious deterministic relation between each population.

the straight line of equation $F=M$ is clearly a mean behaviour, but there is an important scatter aroung this mean behaviour, indicating no deterministic relation. We thus now focus on a stochastic relation between the two series, with the introduction of the ratio $r$, defined as (when $F \neq 0$ ):

$$
r(t)=\frac{M(t)}{F(t)}
$$

This ratio is seen as a new time series, whose statistical properties will be studied here, conditioned on the values of $F$. We first estimate the conditional average of $r$, denoted as $m(x)$ :

$$
m(x)=\langle r(t) \mid F(t)=x\rangle=\int r(t) p(r(t) \mid F(t)=x) d t
$$

$m(x)$ is thus the average value of $r$, estimated for a limited range of values of $F$. For the experimental estimation, the range of values of $F$ (between a minimum $F_{\min }$ and a maximum value $F_{\max }$ ) is divided into a finite number of intervals. The conditional average is then $\left\langle r(t) \mid x-\frac{\Delta x}{2} \leq F(t) \leq x+\frac{\Delta x}{2}\right\rangle$ where $\Delta x$ is the width of the interval considered around position $x$. This has been performed for many intervals bounded by $F_{\min }$ and $F_{\max }$. The result is shown in Fig. 6. We see that when $F$ is larger than 10 individuals $/ \mathrm{m}^{3}$, the mean $m(x)$ of the ratio $r$ is close to 1 , while for $F$ smaller than 10, the mean ratio is larger, reaching values close to 24 for very small abundances.There are 1229 


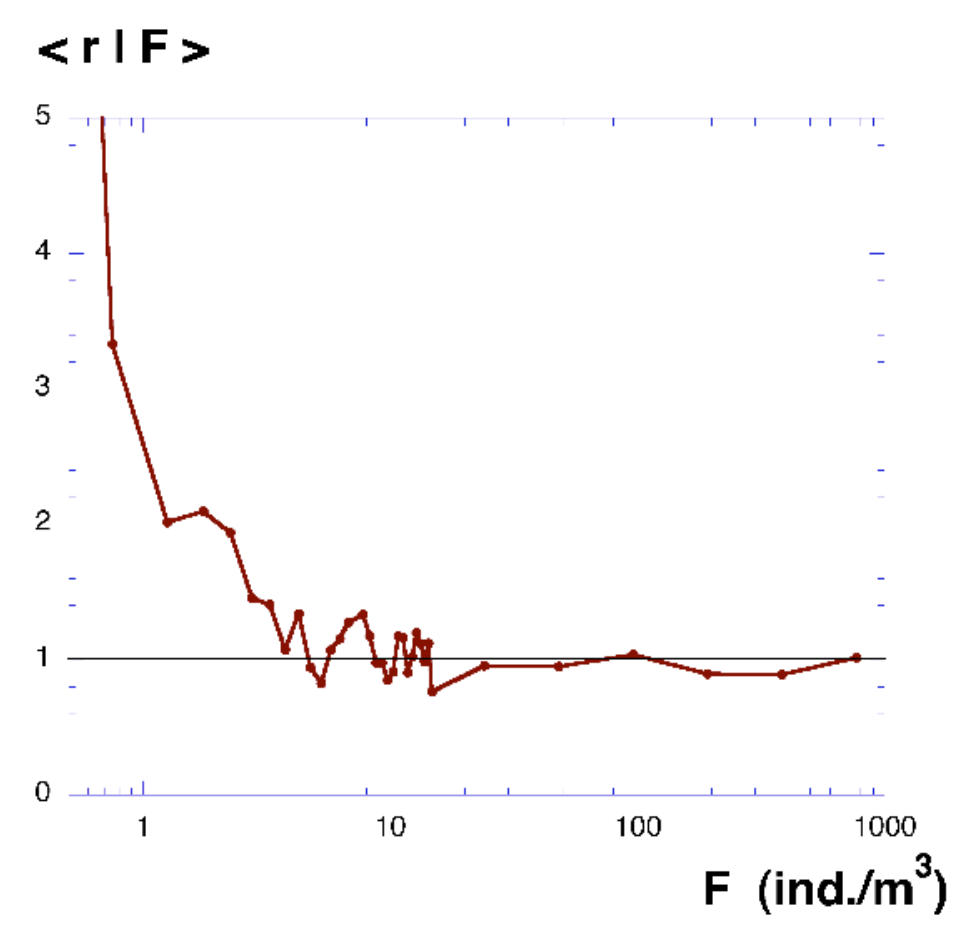

Fig. 6. The conditional average $\langle r \mid F\rangle$ of the ratio $r=M / F$ conditioned on values of the female abundance $F$. When $F$ is larger than 10 individuals $/ \mathrm{m}^{3}$, the ratio is close to 1, while for $F$ smaller than 10, the ratio is larger, reaching values close to 24 for very small abundance.

values for which $r$ can be estimated (when $F \neq 0$ ); on this total population, the mean is $m=\langle r\rangle=1.26$. For the mean conditioned on larger values of $F$, there are 877 values, with the mean given as: $m^{+}=\langle r \mid F \geq 10\rangle=0.97$. For the mean conditioned on smaller values of $F$, there are 352 values, with the mean given as: $m^{-}=\langle r \mid F \leq 10\rangle=1.98$. This numerically confirms the visual information provided by Fig. 6 . In the modelisation of male-female population dynamics, it is then possible to model the male population depending on the female density; for larger female density, the multiplicative factor is a random variable of mean 1, and of pdf given by Fig. 7. For smaller female density values, a new random variable must be chosen, having a mean which increases for smaller values of the female density, as shown by the left-hand side of the curve in Fig. 6.

\subsection{Encounters}

Two of the major aspects of copepod dynamics involve contact rates between particles in turbulent flows: predator-prey encounter rates, and mating encounter rates. The modelization of encounters is thus a critical issue in plankton ecology. Models of encounter rate involve behaviour and turbulence kernels, and the population density. The total encounters between a population 


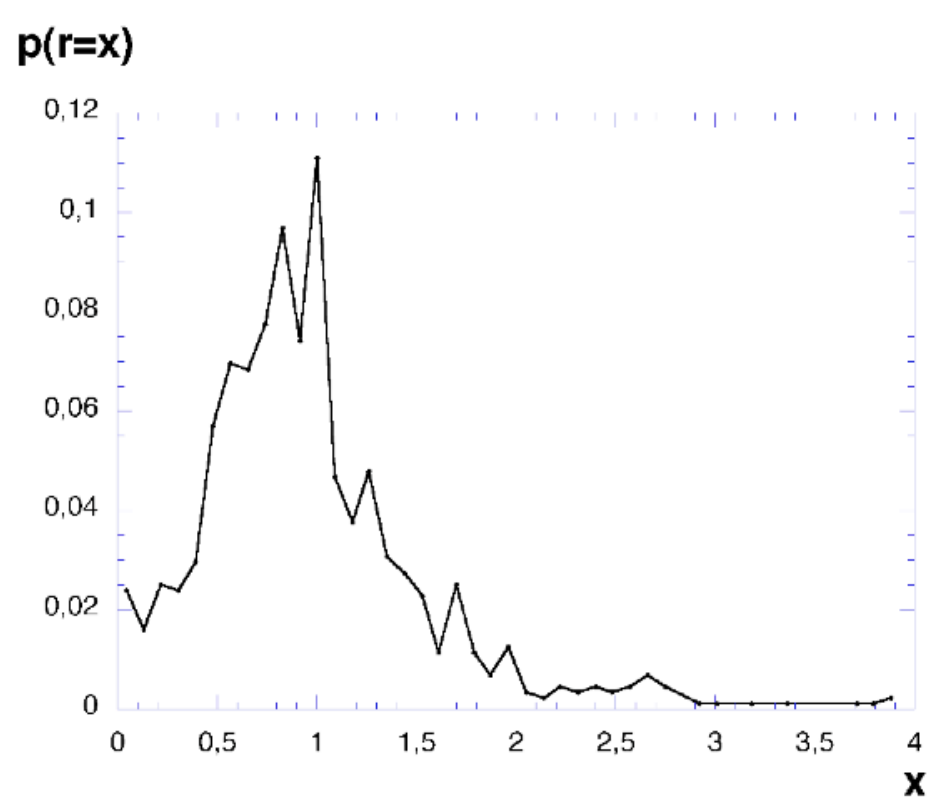

Fig. 7. The conditional pdf of the ratio $r$, estimated for values of female abundance $F$ larger than 10 individuals $/ \mathrm{m}^{3}$.

of $M$ males and $F$ females may be written as:

$$
Z=M F K(\text { turb }, \text { behav })
$$

where $K($ turb, behav) denotes behaviour and turbulence kernels. Here we do not consider these kernels, and study instead the prefactor given by the product $P=M F$ of the densities. We have seen above that the densities are extremely intermittently distributed. We thus expect that the prefactor $P$ is also of high variability. We have estimated this product and its pdf: Fig. 8 shows that it also belongs to power-law distributions; in fact for this field, the power-law is a rather good fit for the whole range of the distribution. The same figure shows the Hill estimator applied to the product time series, and giving the value $\mu=1.25 \pm 0.05$. This index is really small, indicating that the variance of the variable $P$ is not defined.

We notice that the tail index $\mu$ obtained for the product is half the one found in Figs. 3 and 4. We provide here a possible theoretical explanation of this relation. We note that $P=M F=r F^{2}$ and thus its probability can be written as:

$$
\operatorname{Pr}(P \geq x)=\int \operatorname{Pr}\left(F^{2} \geq x / u ; r=u\right) d u
$$

assuming independence for large values of the female abundances, this becomes:

$$
\int \operatorname{Pr}\left(F^{2} \geq x / u\right) \operatorname{Pr}(r=u) d u=\int \operatorname{Pr}(F \geq \sqrt{x / u}) \operatorname{Pr}(r=u) d u
$$

Here since the density of $F$ has a power-law tail of exponent $-\mu-1$, its 


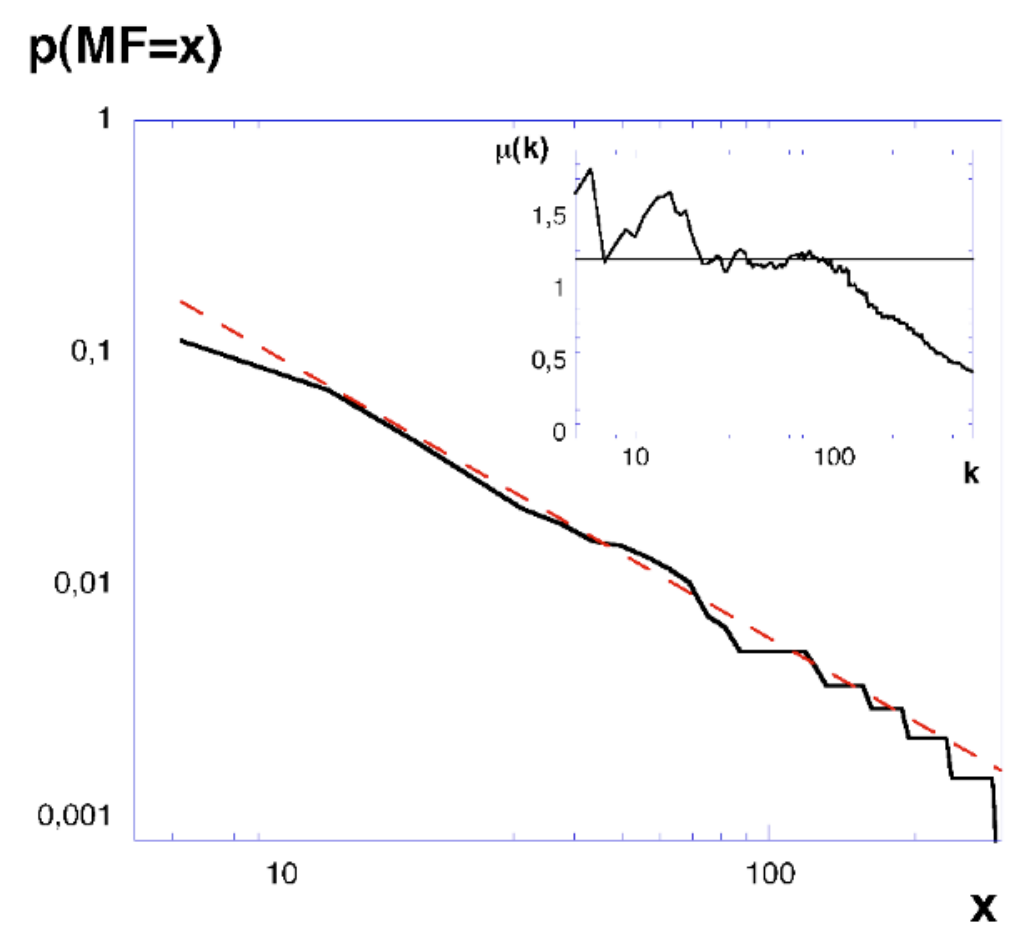

Fig. 8. The pdf of the product $P=M F$ of male and female abundances, in log-log plot, showing an extremely intermittent distribution with extreme events obeying an hyperbolic law of the type $p(x) \sim x^{-\mu-1}$ with $\mu=1.25 \pm 0.05$ as estimated using Hill's estimator (insert).

probability distribution has a tail $-\mu$, and:

$$
\operatorname{Pr}(F \geq \sqrt{x / u}) \sim\left(\frac{x}{u}\right)^{-\mu / 2} \quad ; \frac{x}{u} \gg 1
$$

Equations (9) and (10) finally give:

$$
\operatorname{Pr}(P \geq x) \sim x^{-\mu / 2}\left\langle r^{\mu / 2}\right\rangle \sim x^{-\mu / 2} \quad ; x \gg 1
$$

This shows that the pdf of the product is also a power-law, with a characteristic exponent of $\mu / 2$.

\section{Discussion}

The investigation of extreme events has numerous implications in ecology since they shape the physiology, ecology and evolution of organisms. Some relevant examples in ecology are provided by HABs, microphytobenthos patches when power pdfs have been found for spatial fluctuations [18], large-fire disruptions, extinctions of populations [8]. And recently an increasing awareness exists on 
extreme events in marine ecosystems which have occurred across distinct regions and over a large spectrum of marine food webs, and seem to be related to climate fluctuations. The approach we used in this work shows that understanding the dynamics of extreme events constitutes a powerful tool to model not only the frequency but also the severity of such events. Furthermore, these results have implications for the modeling of the population growth but also the dynamics of the encounters between sexes, which drives mating systems. This is important since for populations of species that reproduces sexually the population growth may be constrained by encounters [17].

Although the approach used was applied on a single species, the procedure may be used to investigate extreme events in other populations, such as gelatinous carnivores, harmful algal blooms, whose incidence may constitute public health risks and may have an effect on losses of fisheries activities, as well as on tourism.

Intermittency is a well-known phenomenon in various fields of physics and occurs frequently in biological time-series. Hence, it should be taken into account when studying the dynamics of populations. Here we have shown that once the dynamics and properties of extremes have been identified the population can be described by a simple reduced-order model involving few parameters. Subsequently, these models may have numerous applications, because extremes in population densities are often associated with substantial consequences in the population dynamics (e.g. outbreaks, drops). A further step should investigate whether extreme events in ecological time series are driven by the intrinsic population dynamics or related to extreme events in physical forcing.

Another possible application of these results is that they stress a potential selective pressure which may vary at high and low density populations, as showed by the strong deviation from $50: 50$ ratio of males females. At small female density $\left(<10\right.$ ind. $\left./ \mathrm{m}^{3}\right)$ the males density increases. From the ecological point of view, the low female densities may reduce the population growth because of the small population size, the so-called Allee effect [19], and the higher number of males may enhance the encounter matings between sexes. This is likely a challenge the population must deal to avoid the risk of extinction, and more generally this is a central question in ecology: understanding how competition for resources changes individual fitness at low compared to high densities [20-22].

Overall, for long time little attention has been given to extremes events in marine ecology, even though they strongly influence the functioning of ecosystems (i.e. pests, extinctions, regime shifts). The statistics of such events offer a potential advantage to scale their properties and hence to incorporate them into forecast studies. 


\section{Acknowledgements}

The authors are particularly grateful to Serge Dallot whose commitment to the plankton monitoring in the Villefranche Bay has made possible this longterm series. This paper is a contribution to the IFB "Institut Franais de la Biodiversite" project "Biodiversité et changement global".

\section{References}

[1] S. L. Pimm and A. Redfearn, The variability of population dynamics, Nature 334 (1988) 613-614.

[2] A. Blarer and M. Doebeli, Resonance effects and outbreaks in ecological time series, Ecology Letters 2 (1999) 167-177.

[3] P. Inchausti and J. Halley, Investigating long-term ecological variability using the Global Population Dynamics database, Science 293 (2001) 655-657.

[4] M. Pascual and F. Guichard, Criticality and disturbance in spatial ecological systems, Trends Ecol. Evol. 20 (2005) 88-95.

[5] S. D. Gaines and M. W. Denny, The largest, smallest, highest, lowest, longest, and shortest: extremes in ecology, Ecology 74 (1993) 1677-1692.

[6] A. Arino and S. L. Pimm, On the nature of population extremes, Evol. Ecology 9 (1995) 429-443.

[7] R. Ferriere and B. Cazelles, Universal power laws govern intermittent rarity in communities of interacting species, Ecology 80 (1999) 1505-1521.

[8] R. W. Katz and G. S. Brush and M. B. Parlange, Statistics of extremes: modeling ecological disturbances, Ecology 86 (2005) 1124-1134.

[9] M. D. Ohman and H. J. Hirche, Density-dependent mortality in an oceanic copepod population, Nature 412 (2001) 638-641.

[10] J. D. Reynolds and R. P. Freckleton, Population dynamics: growing to extremes, Science 309 (2005) 567-568.

[11] M. R. Leadbetter and G. Lindgren and H. Rootzen, Extremes; and related properties of random sequences and processes, Springer, New York, 1983.

[12] M. Mitzenmacher, A brief history of generative models for power law and lognormal distributions, Internet Mathematics 1 (2003) 226-251.

[13] M. E. J. Newman, Power laws, Pareto distributions and Zipf's law, Contemp. Phys. 46 (2005) 323-350. 
[14] B. M. Hill, A simple and general approach to inference about the tail of a distribution, Ann. Statist. 3 (1975) 1163-1174.

[15] J. Caers and J. Beirlant and P. Vynckier, Bootsrap confidence intervals for tail indices, Comp. Statist. Data Analy. 26 (1998) 259-277.

[16] L. Peng, Asymptotically unbiased estimators for the extreme-value index, Statist. Prob. Lett. 38 (1998) 107-115.

[17] F. G. Schmitt and C. Nicolis, Scaling of return times for a high resolution rainfall time series, Fractals 10 (2002) 285-290.

[18] L. Seuront and N. Spilmont, Self-organized criticality in intertidal microphytobenthos patch patterns, Physica A 313 (2002) 513-539.

[19] W. C. Allee and A. E. Emerson and O. Park and T. Park and K. P. Schmidt, Principles of Animal Ecology, Saunders, Philadelphia, 1949.

[20] A. Grant, Selection pressures on vital rates in density dependent populations, Proc. R. Soc. B 264 (1997) 303-306.

[21] N. L. Rodenhouse and T. W. Sherry and R. T. Holmes, Site-dependent regulation of population size: a new synthesis, Ecology 78 (1997) 2025-2042.

[22] W. J. Sutherland and K. Norris, Behavioural models of population growth rates: implications for conservation and prediction, Phil. Trans. R. Soc. B 357, (2002) 1273-1284. 